\section{Sarcoglycan complex in masseter and sternocleidomas- toid muscles of baboons: an immunohistochemical study}

\author{
G. Cutroneo, ${ }^{1}$ A. Centofanti, ${ }^{1}$ F. Speciale, ${ }^{1}$ \\ G. Rizzo, ${ }^{1}$ A. Favaloro, ${ }^{1}$ G. Santoro, ${ }^{1}$ \\ D. Bruschetta, ${ }^{1}$ D. Milardi, ${ }^{1,2}$ A. Micali, ${ }^{1}$ \\ D. Di Mauro, ${ }^{1}$ G. Vermiglio, ${ }^{1}$ G. Anastasi, ${ }^{1}$ \\ F. Trimarchi ${ }^{1}$
}

'Dipartimento di Scienze Biomediche e delle Immagini Morfologiche e Funzionali, Università di Messina

${ }^{2}$ IRCCS Centro Neurolesi Bonino-Pulejo, Messina, Italy

\begin{abstract}
The sarcoglycan complex consists of a group of single-pass transmembrane glycoproteins that are essential to maintain the integrity of muscle membranes. Any mutation in each sarcoglycan gene causes a series of recessive autosomal dystrophin-positive muscular dystrophies. Negative fibres for sarcoglycans have never been found in healthy humans and animals. In this study, we have investigated whether the social ranking has an influence on the expression of sarcoglycans in the skeletal muscles of healthy baboons. Biopsies of masseter and sternocleidomastoid muscles were processed for confocal immunohistochemical detection of sarcoglycans. Our findings showed that baboons from different social rankings exhibited different sarcoglycan expression profiles. While in dominant baboons almost all muscles were stained for sarcoglycans, only $55 \%$ of muscle fibres showed a significant staining. This different expression pattern is likely to be due to the living conditions of these primates. Sarcoglycans which play a key role in muscle activity by controlling contractile forces may influence the phenotype of muscle fibres, thus determining an adaptation to functional conditions. We hypothesize that this intraspecies variation reflects an epigenetic modification of the muscular protein network that allows baboons to adapt progressively to a different social status.
\end{abstract}

\section{Introduction}

The dystrophin-glycoprotein complex (DGC) is composed of several transmembrane and peripheral proteins and is highly expressed in the sarcolemma of skeletal muscles. ${ }^{1,2} \alpha$-dystroglycan is a central protein in the sarcolem- ma that binds to ligands in the surrounding basal lamina through $\beta$-dystroglycan. ${ }^{3}$ Dystrophin, in turn, binds to the sub-membrane actin and the intermediate filament cytoskeleton, thus completing the link between the cytoskeleton and the extracellular matrix. ${ }^{4}$ The sarcoglycan complex (SGC) in the DGC consists of six subunits of single-pass transmembrane glycoprotein sub-units named $\alpha$-, $\beta-, \gamma-, \delta$-, $\varepsilon$-, and $\zeta$-sarcoglycan (SG). The SGC is linked to dystrophin by a lateral connection with the dystroglycan complex, ${ }^{5-7}$ and is in turn formed by six distinct sub-units. Therefore, the localization of the DGC in the costameres and the identification of its function as a link between the matrix and the cytoskeleton has led to hypothesize that the DGC, and particularly the SGC, might be critical in stabilizing muscles and their sarcolemma during contraction and relaxation. ${ }^{3,8}$ In addition, some experimental evidence demonstrated that SGs may be involved directly in L6 cell adhesion by interacting with integrins, thus revealing the presence of a bidirectional signalling pathway between SGs and the integrin adhesion system. ${ }^{9,10}$ This bidirectional interaction could be crucial in the regulation of the formation of the adhesion system. ${ }^{9}$

The role of SGs and integrins was demonstrated in muscle tissues both in normal and pathological conditions. ${ }^{2,3,10-12}$ Mutations in dystrophin or SGs are associated with a decreased expression or incomplete formation of the entire DGC ${ }^{10,13}$ resulting in several muscular diseases such as Duchenne and Becker muscular dystrophies and Limb Girdle Muscular Dystrophy respectively. ${ }^{14-17}$ Moreover, other investigations demonstrated that the enhanced expression of $\alpha 7 \beta 1$ attenuates muscular dystrophy and restores viability in dystrophic mice. ${ }^{18}$ It is clear that DGC components, and in particular sarcoglycans, play a key role in the function of the muscle fibres that are necessary to maintain muscular viability. ${ }^{12,19}$ Indeed, negative fibres in the DGC have never been found in healthy humans and animals.

We have recently analysed integrins in masseter muscle fibres in a malocclusion and showed that these proteins play a key role in this muscle, since they regulate the functional activity of muscle fibres and promote the optimization of contractile forces..$^{20}$ Furthermore, in a previous study, we analysed integrins and SGs in the masseter of chimpanzees in order to understand the actual differences between modern humans and these primates, because, as is well-known, humans and chimpanzees do share an identical inversion in chromosomes 7 and 9 that cannot be found in other primates. In particular, we analysed alpha-male and nonalpha male subjects and identified substantial differences in integrin staining patterns. This
Correspondence: Giuseppina Rizzo, Dipartimento di Scienze Biomediche e delle Immagini Morfologiche e Funzionali, Università di Messina, Policlinico Universitario, Torre Biologica, Via Consolare Valeria 1, 98125 Messina, Italy.

Tel. +39.090.2217141 - Fax: +39.090 .692449 .

E-mail: rizzog@unime.it

Key words: Sarcoglycan, baboons, immunohistochemistry, evolution, ranking.

Contributions: GC, GA, study design; GA, GC, AC, FS, GR, GV, experimental procedures, immunohistochemical data processing. AF, GS, AM, DB, collection of biopsies. AF, GV, FT, DDM, DM, microscopic analysis, data collection and/or preparation of figures. All authors reviewed and contributed to the various draft versions of the manuscript and read and approved the final manuscript.

Conflict of interest: the authors declare no conflict of interest.

Acknowledgments: the authors wish to thank the Department of Orthodontics, in particular Head Prof. Felice Festa for his excellent scientific contribution, and the ZooSafari Biopark of Selva di Fasano, led by Scientific Head Dr. Fabio Rausa, for providing the baboons for our study.

Received for publication: 7 February 2015.

Accepted for publication: 21 April 2015.

This work is licensed under a Creative Commons Attribution NonCommercial 3.0 License (CC BYNC 3.0).

(C) Copyright G. Cutroneo et al., 2015 Licensee PAGEPress, Italy

European Journal of Histochemistry 2015; 59:2509 doi:10.4081/ejh.2015.2509

finding led us to hypothesize that SGs, as well as integrins, could participate in the maintenance of contractile forces and in vital biological processes, such as the preservation of tissue integrity, cell differentiation and cellextracellular matrix interactions. ${ }^{21}$ The masseter is an important muscle in primates, because it is used to show dominant and defensive attitudes. For these reasons most primates have powerful masticatory muscles, which, on the contrary, are considerably smaller in modern humans. ${ }^{22}$

In this work, we aim to investigate the behaviour of SGs in skeletal muscles of healthy baboons by analysing high-, middle-, and lowranking subjects. These primates are genetically closer to man than any other Old World monkeys, since their DNA sequence differs only by approximately $4 \%,{ }^{23}$ unlike macaques that have a $6.46 \%$ difference. ${ }^{24}$ In particular, besides studying the masseter muscle, which we had already analysed in previous studies, in 
this case we also focused on the sternocleidomastoid muscle. This muscle, unlike the masseter muscle, derives embryologically from myotomes and is responsible for the control of head movements. As such, it is a very important anatomical component for high-ranking baboons, which are constantly alert to be able to control the group and defend its members.

\section{Materials and Methods}

\section{Tissue samples}

In this study we examined biopsies from masseter and sternocleidomastoid muscles of baboons belonging to different social ranks. In particular, we selected 10 high-ranking adult males (31-39 year-old), 10 middle-ranking adult males (32-39 year-old), and 10 low-ranking adult males (31-40 year-old). All animals were healthy and unaffected by any motor disease. The selected baboons were part of a larger social group (108 animals) living in an
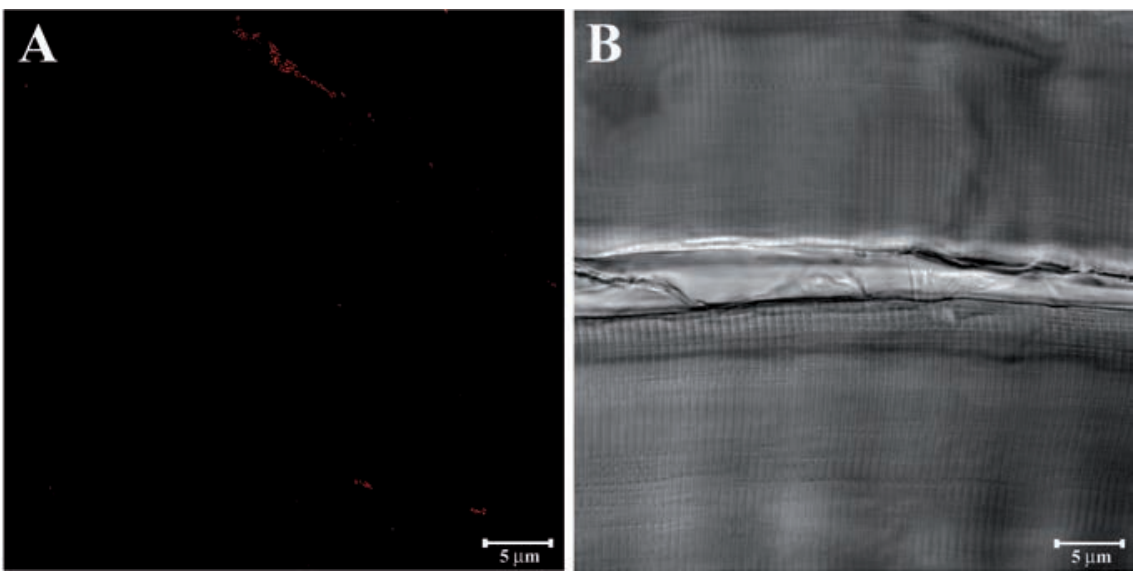

Figure 1. Longitudinal section of baboon masseter muscle immunolabeled only with the secondary antibody $(\mathrm{A})$ and the corresponding transmitted light $(\mathrm{B})$.
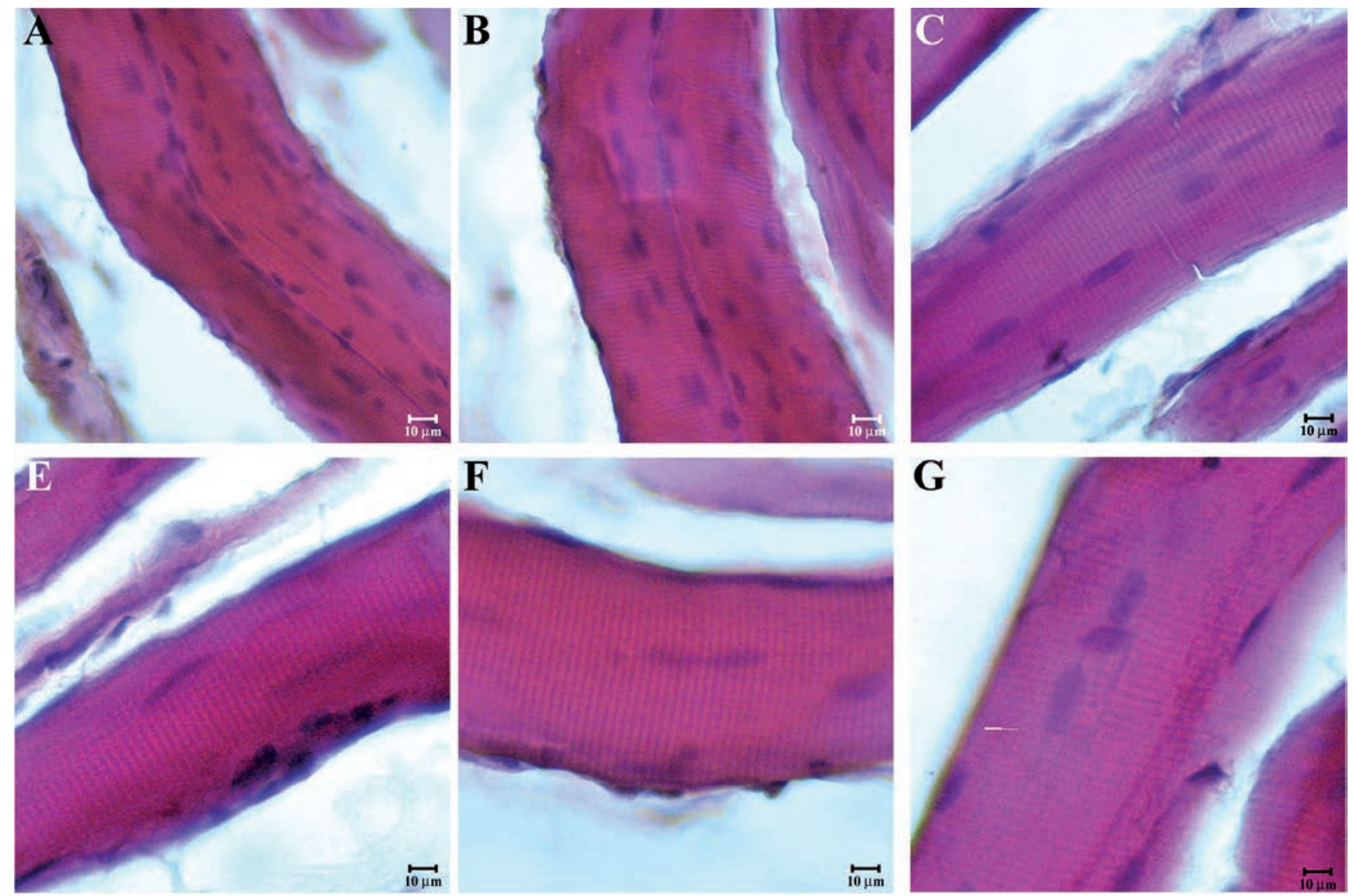

Figure 2. Compound panel showing longitudinal sections of masseter muscle in high- (A), middle- (B), and low-ranking (C) baboons and longitudinal sections of sternocleidomastoid muscle in high- (D), middle- (E), and low-ranking (F) baboons. All sections were stained with the hematoxylin and eosin method and they show a normal morphology of all fibres excluding any disease in the animals enrolled in this study. 
indoor/outdoor enclosure at the Zoosafari Biopark of Fasano (Brindisi, Italy).

Muscle biopsies were performed under anaesthesia. The animals were immobilized by a low dose of diazepam and, subsequently, were anaesthetized with ketamine. After a skin incision to expose the muscle, the specimens were harvested with a $6.0 \mathrm{~mm}$ biopsy punch. Lidocaine was then sprayed as local anaesthetic for pain management.

The baboons were managed in compliance with the recommendations of the institutional and national guide for the care and use of laboratory animals.

\section{Light microscopy}

The specimens were fixed overnight in 2.5\% glutaraldehyde mixed with $4 \%$ paraformaldehyde in a $0.1 \mathrm{M}$ sodium cacodylate buffer at $4^{\circ} \mathrm{C}$, dehydrated in ethanol, and infiltrated with Technovit 9100 . Seven- $\mu$ m sections were cut with a LKB Ultrotome V (LKB Instruments, Mt Waverley, Victoria, Australia), and counterstained with hematoxylin (10 min) and eosin (5 min). Then, the sections were examined and photographed with a Primo Star light microscope (Carl Zeiss, Jena, Germany).

\section{Immunohistochemistry}

Biopsies were fixed in $3 \%$ paraformaldehyde in a $0.2 \mathrm{M}$ phosphate buffer with a $\mathrm{pH}$ of 7.4 for $2 \mathrm{~h}$ at room temperature. After numerous rinses in a $0.2 \mathrm{M}$ phosphate buffer with a $\mathrm{pH}$ of 7.4, and phosphate-buffered saline (PBS), the biopsies were infiltrated with $12-18 \%$ sucrose. They were then snap-frozen in liquid nitrogen. Some 20- m thick sections were cut with a cryostat and placed on glass slides coated with $0.5 \%$ gelatine and $0.005 \%$ chromium potassium sulphate. In order to block nonspecific binding sites and permeabilize membranes, the sections were pre-incubated with $1 \%$ bovine serum albumin, $0.3 \%$ Triton X-100 in PBS, for $15 \mathrm{~min}$, at room temperature. Finally, the sections were incubated with primary antibodies. The following primary goat polyclonal antibodies were used with a 1:100 dilution: anti- $\alpha-S G$, anti- $\beta-S G$, anti- $\gamma-S G$, anti- $\delta-S G$, anti- $\varepsilon-S G$, and anti- $\zeta-S G$ (Santa Cruz Biotechnology, Santa Cruz, CA, USA). Primary antibodies were detected using Texas Red-conjugated antigoat IgG (Jackson ImmunoResearch Laboratories, West Grove, PA, USA).

Negative control staining was performed following the same procedure, except that the primary antibody was omitted or replaced by non-immunized goat serum.

Sections were then analysed and images captured with a Zeiss LSM 5 DU0 (Carl Zeiss) confocal laser scanning microscope. All images were digitalized with an 8-bit resolution into an array of 2048 x 2048 pixels. Optical sections of fluorescence specimens were obtained using a HeNe laser (543 nm) at a 1-min, 2-sec scanning speed with up to eight averages and 1.50 -mm-thick sections of fluorescent specimens were obtained using a pinhole of 250 . Contrast and brightness were measured by examining the brightest labelled pixels and choosing the settings that allowed a clear visualization of structural details, while keeping the highest pixel intensities close to 200 . The same settings were used for all images obtained from the other samples that were
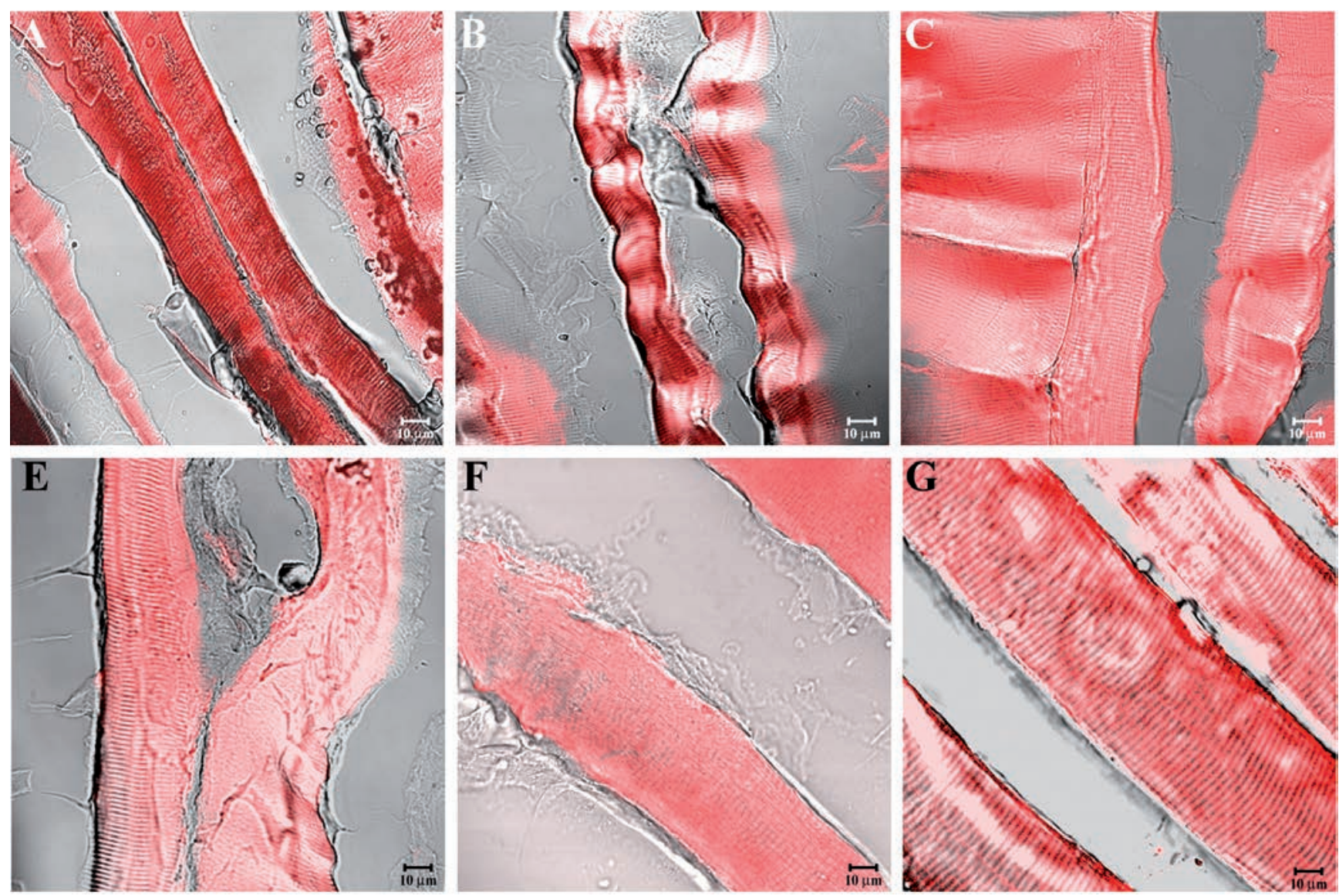

Figure 3. Compound panel showing immunohistochemical findings from masseter muscles of high-ranking baboons. The samples were immunolabelled with antibodies against $\alpha$-SG (A), $\beta$-SG (B), $\gamma$-SG (C), $\delta$-SG (D), $\varepsilon-S G(E)$, and $\zeta-S G(F)$. All tested proteins showed a normal and clear staining pattern in all fibres. 
processed in parallel. For each reaction, at least 100 individual fibres were examined.

For image analysis, we used the splitting function that shows individual channels and relative merge and the acquisition of a Z-stack to analyse the staining pattern across the sections. Digital images were cropped and figure montages were prepared using Adobe Photoshop 7.0 (Adobe System, Palo Alto, CA, USA).

\section{Results}

In this study, we analysed the immunestaining patterns of $\alpha$-SG, $\beta$-SG, $\gamma$-SG, $\delta$-SG, $\varepsilon$ SG and $\zeta-S G$, using specific antibodies. Initially, we performed a negative control on skeletal muscle samples of baboons by omitting the primary antibody (Figure 1A). In Figure 1B, we show the corresponding transmitted light image of Figure 1A. In order to demonstrate that the tested muscle fibres were healthy, the samples were stained with the hematoxylin and eosin method; the normal arrangements and morphologies of masseter muscle fibres in high- (Figure 2A), middle(Figure 2B), and low-ranking baboons (Figure $2 \mathrm{C})$ rule out any disease in the animals selected for this study. Similar morphology has been observed for sternocleidomastoid muscle in high- (Figure 2D), middle (Figure 2E), and low-ranking baboons (Figure 2F). Immunofluorescence reactions on the masseter muscle of high-ranking baboons showed that $\alpha$-SG (Figure 3A), $\beta$-SG (Figure 3B), $\gamma$-SG (Figure 3C), $\delta$-SG (Figure 3D), $\varepsilon$-SG (Figure $3 \mathrm{E}$ ), and $\zeta$-SG (Figure 3F) revealed a clear staining pattern across the fibres. The merger between transmitted and red fluorescent light demonstrated that all fibres were labelled. A similar result was obtained for all SGs analysed in sternocleidomastoid muscle fibres (Figure 4 A-F). In masseter muscle samples obtained from middle-ranking baboons, immunofluorescence for $\alpha$-SG (Figure 5A), $\beta$ SG (Figure 5B), $\gamma$-SG (Figure 5C), $\delta$-SG (Figure 5D), $\varepsilon$-SG (Figure 5E), and $\zeta-S G$ (Figure 5F) showed that most fibres were reactive for the tested proteins, while only a few fibres (approximately 15\%) revealed a weak (yellow arrows in Figure 5C) or no (yellow arrowheads in Figure 5F) fluorescence. Results were similar for the sternocleidomastoid muscle (Figure 6A-F). The split of some previous images showed a weak or absent staining pattern of SGs in the masseter (yellow arrow in Figure $7 \mathrm{~A}-\mathrm{C}$ ) and sternocleidomastoid (yellow arrowhead in Figure 7 D-F) muscles. Immunofluorescence reactions with antibodies against $\alpha$-SG (Figure 8A), $\beta$-SG (Figure $8 \mathrm{~B}), \gamma$-SG (Figure 8C), $\delta$-SG (Figure 8D), $\varepsilon$-SG (Figure 8E), and $\zeta$-SG (Figure 8F) on the masseter muscle obtained from low-ranking baboons showed several fibres (approximately $55 \%$ ) with no staining pattern for the tested proteins (yellow arrows). Similarly, in the sternocleidomastoid muscle, several fibres showed no staining pattern for SGs (Figure 9 A-F).

We used a 3-dimensional reconstruction to analyse the entire thickness of the sections $(16 \mu \mathrm{m})$, even though we eliminated the first 2 and last 2 sections, because immunofluorescence was not detectable. This application allowed us to obtain a gallery of 16 sections, each with a scan step size of $1 \mu \mathrm{m}$ in a single localization reaction. This reconstruction
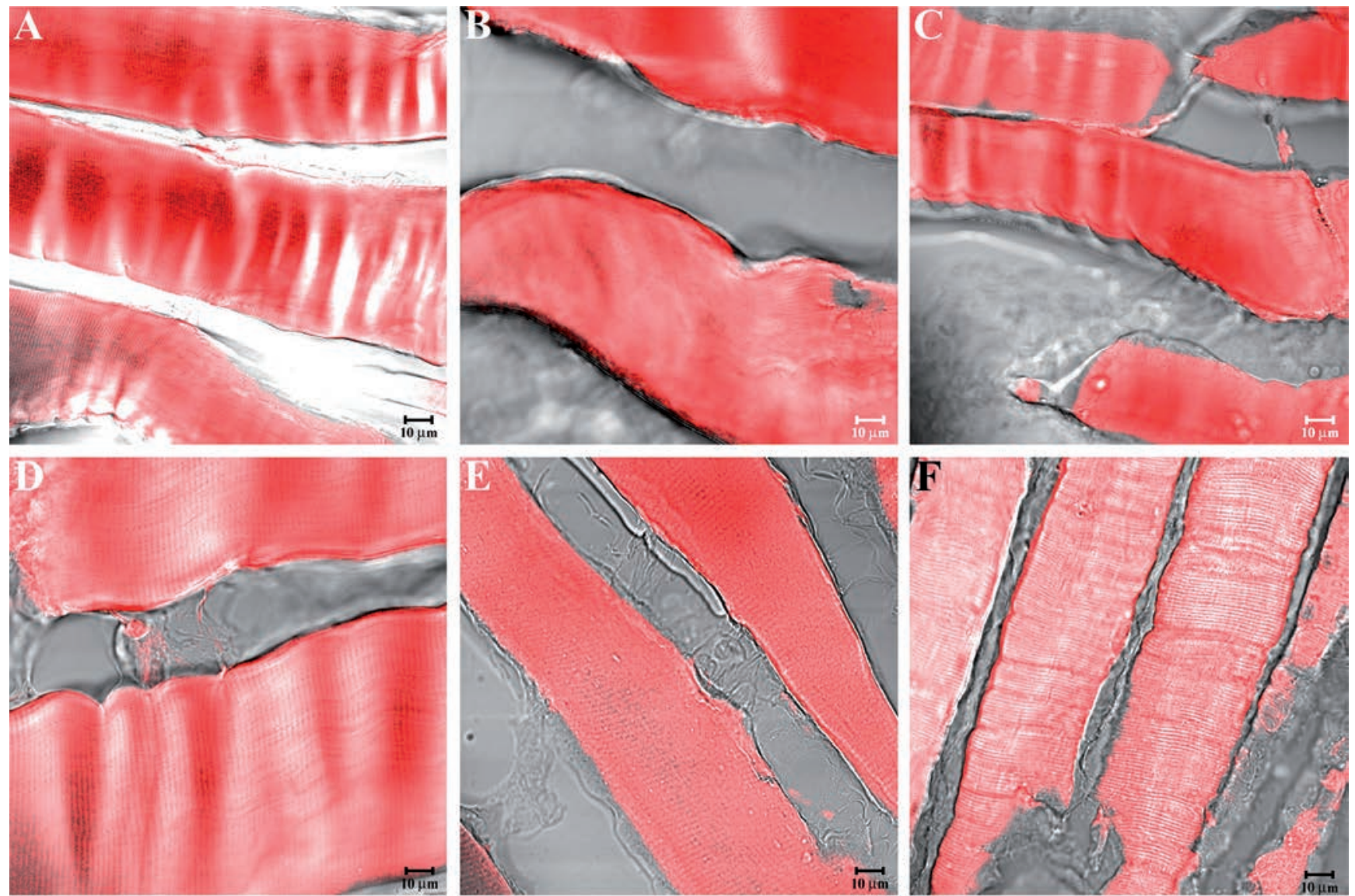

Figure 4. Compound panel showing immunohistochemical findings from sternocleidomastoid muscles of high-ranking baboons. The samples were immunolabelled with antibodies against $\alpha$-SG (A), $\beta$-SG (B), $\gamma$-SG (C), $\delta$-SG (D), $\varepsilon-S G(E)$, and $\zeta-S G(F)$. All tested proteins showed a normal and clear staining pattern in all fibres. 

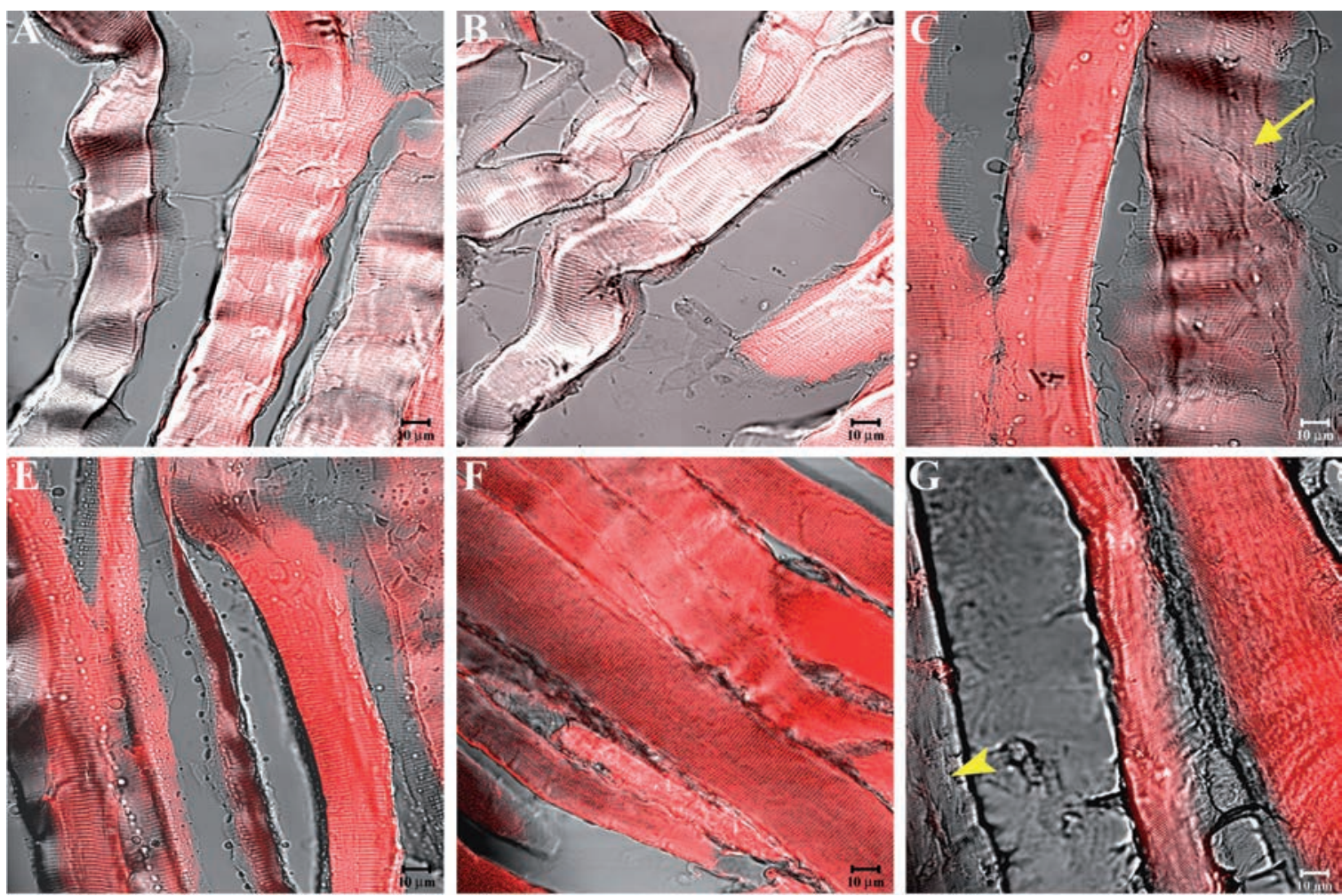

Figure 5. Compound panel showing immunohistochemical findings from masseter muscles of middle-ranking baboons. The samples were immunolabelled with antibodies against $\alpha$-SG (A), $\beta$-SG (B), $\gamma$-SG (C), $\delta$-SG (D), $\varepsilon-S G(E)$, and $\zeta-S G(F)$. Most fibres present a clear staining pattern for the tested proteins, while a few fibres display weak (yellow arrows in $\mathrm{C}$ ) or no (yellow arrowheads in $\mathrm{F}$ ) fluorescence.
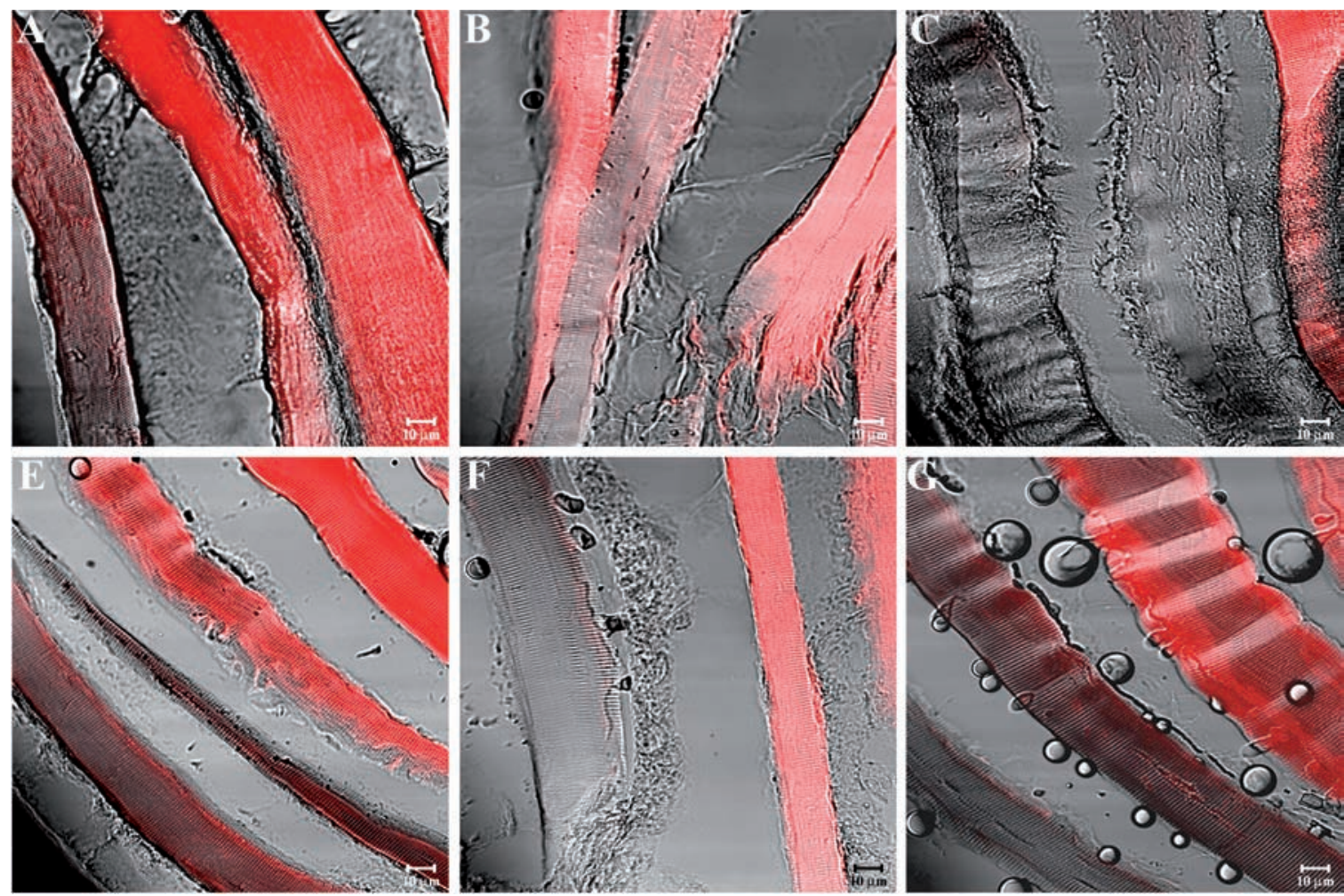

Figure 6. Compound panel showing immunohistochemical findings from sternocleidomastoid muscles of middle-ranking baboons. The samples were immunolabelled with antibodies against $\alpha$-SG (A), $\beta$-SG (B), $\gamma$-SG (C), $\delta$-SG (D), $\varepsilon-S G(E)$, and $\zeta$-SG (F). Most fibres present a clear staining pattern for the tested proteins, while a few fibres display weak or no fluorescence. 

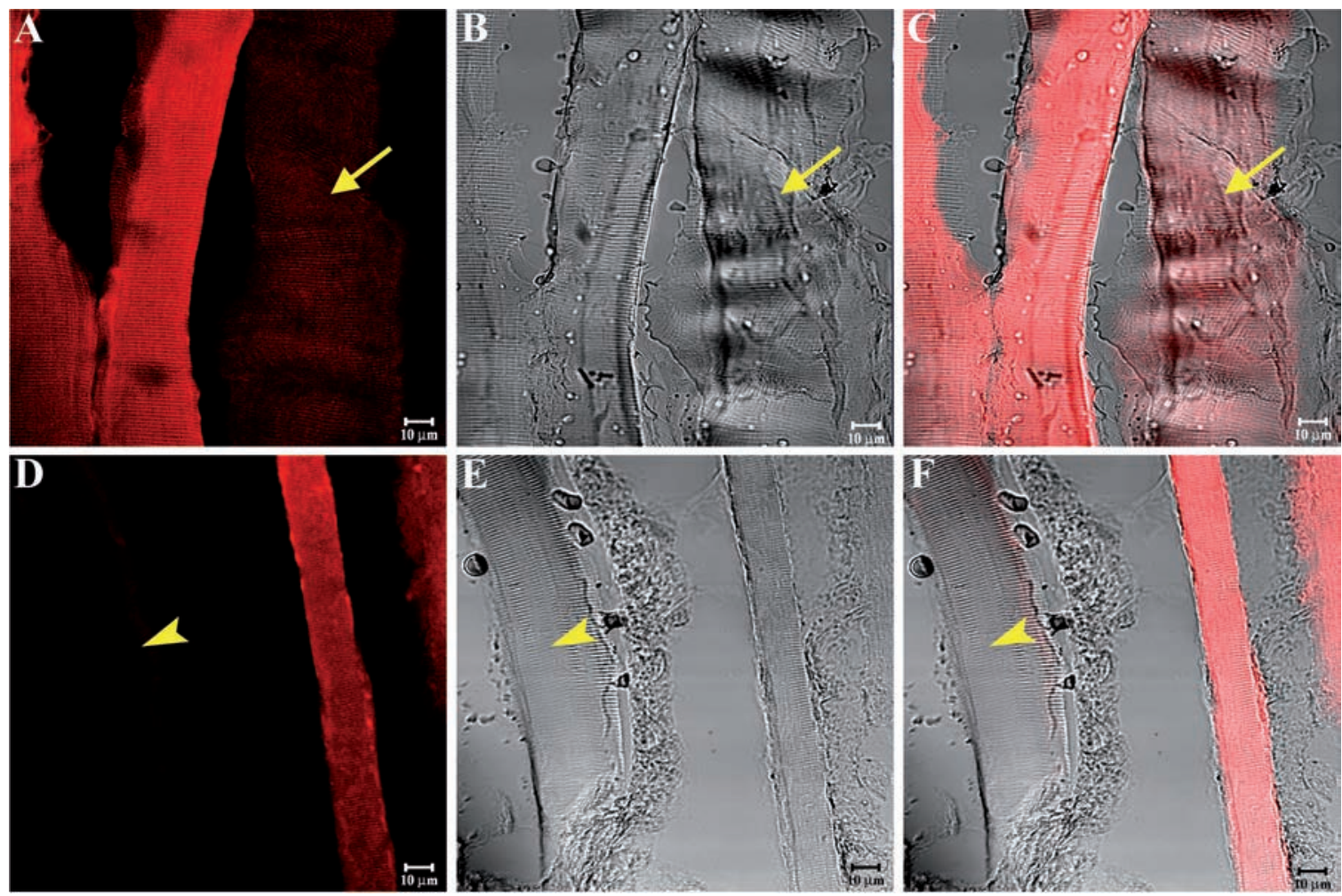

Figure 7. Compound panel showing a split of the immunohistochemical reactions reported in Figure 5C (A-C) and in Figure 6E (D-F). This function highlights more clearly a weak staining pattern (yellow arrow in A-C) and an absent staining pattern (yellow arrowhead in D-F) in some fibres of middle-ranking baboons.
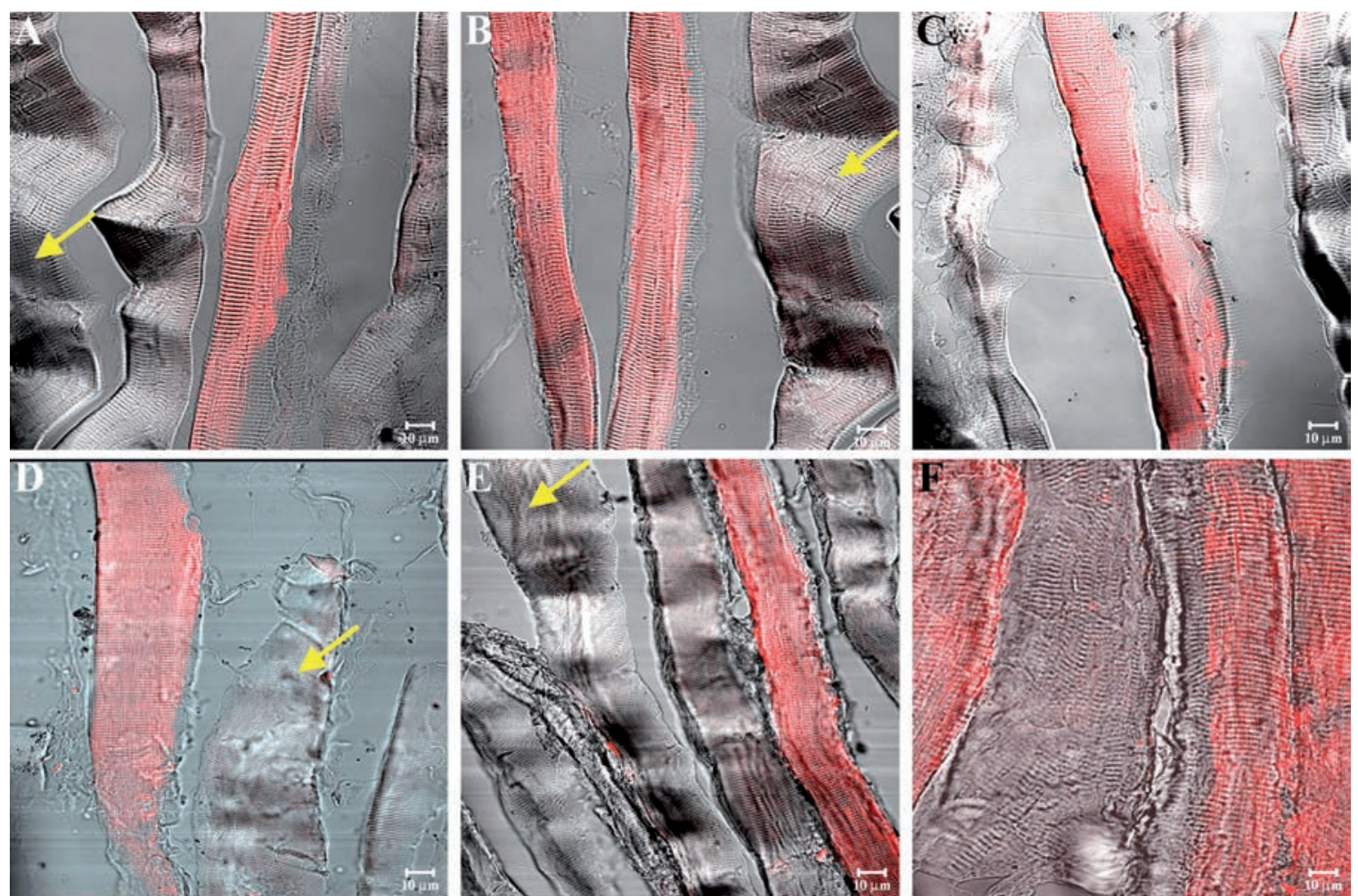

Figure 8. Compound panel showing immunohistochemical findings from masseter muscles of low-ranking baboons. The samples were immunolabelled with antibodies against $\alpha$-SG (A), $\beta$-SG (B), $\gamma$-SG (C), $\delta$-SG (D), $\varepsilon$-SG (E), and $\zeta-S G(F)$. Several fibres showed no staining pattern for SGs (yellow arrows). 

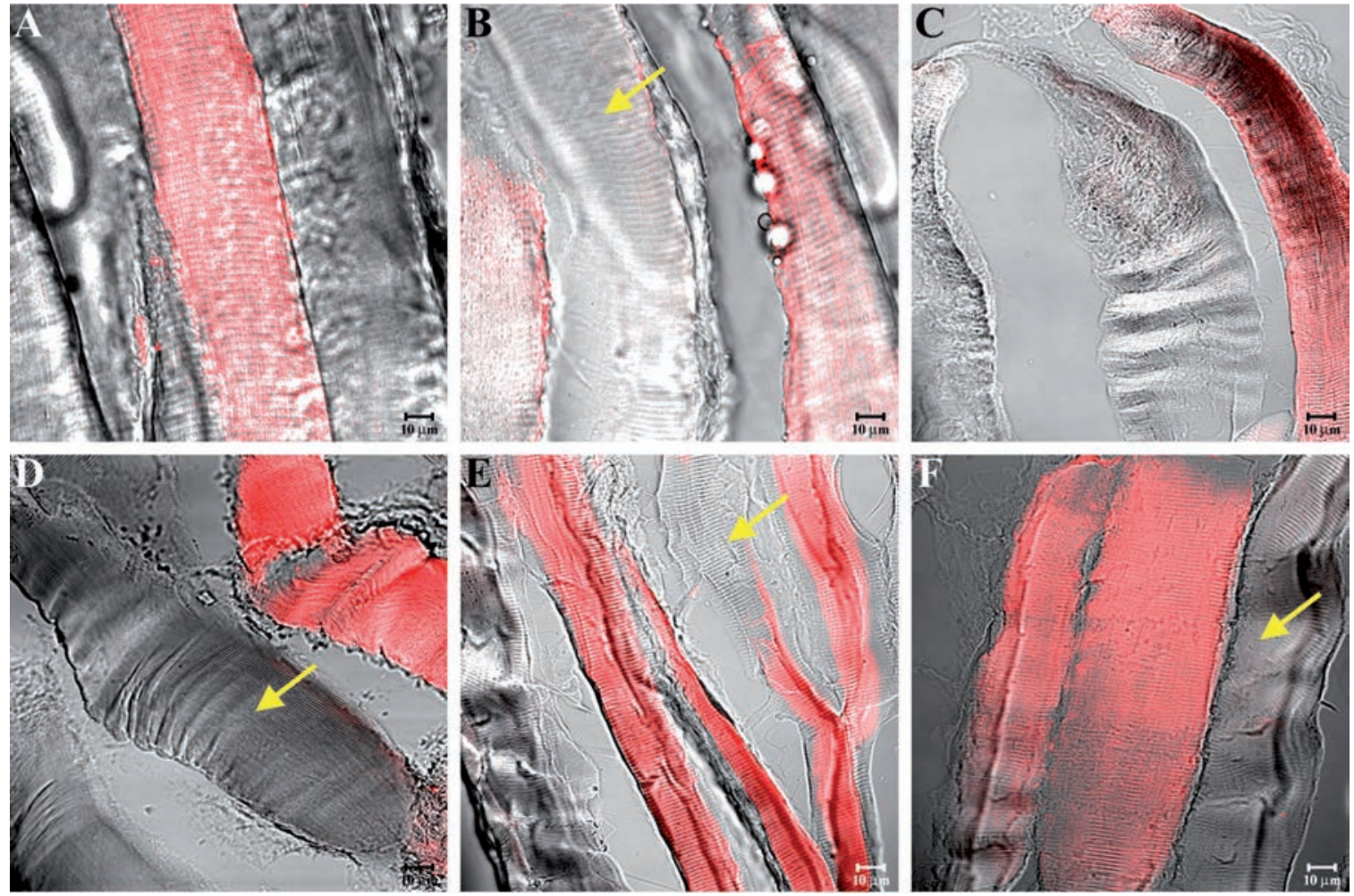

Figure 9. Compound panel showing immunohistochemical findings from sternocleidomastoid muscles of low-ranking baboons. The samples were immunolabelled with antibodies against $\alpha$-SG (A), $\beta$-SG (B), $\gamma$-SG (C), $\delta$-SG (D), $\varepsilon$-SG (E), and $\zeta$-SG (F). Several fibres showed no staining pattern for SGs (yellow arrows).

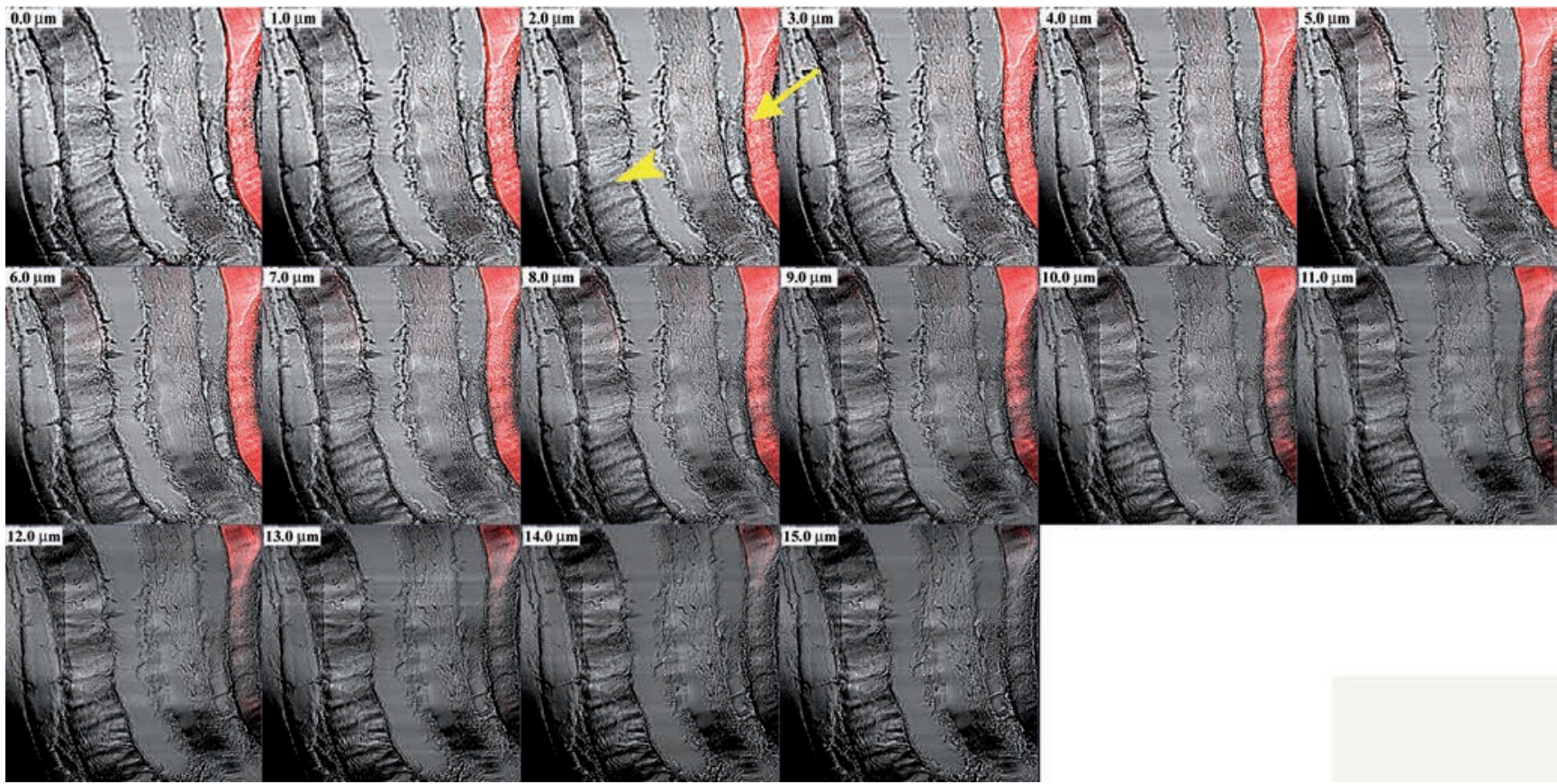

Figure 10. Gallery of 16 sections, each with a scan step of $1.0 \mu \mathrm{m}$, showing a 3-dimensional reconstruction of the entire thickness of the sections, displayed in Figure 6C, immunolabelled with antibody against $\gamma-S G$ to focus and analyse the entire fibre. The staining pattern, for $\gamma$-SG is present (arrow) or absent (arrowhead) across the entire thickness of the sections, while fibres are always visible. 
showed that in this case the staining pattern for $\gamma$-SG of the sternocleidomastoid muscle obtained from middle-ranking baboons is present (arrow) or absent (arrowhead) across the entire sections, despite fibres are always visible (Figure 10).

\section{Discussion}

The sarcoglycan complex, like adhesion focal integrins, binds the actin cytoskeleton with the extracellular matrix..$^{13}$ An appropriate maintenance of this link is thought to be important to ensure the mechanical viability of the sarcolemma. ${ }^{3}$

In this study, we have analysed for the first time SGs using samples of masseter and sternocleidomastoid muscles from healthy high-, middle-, and low-ranking baboons to understand whether these proteins can play a key role in contractile forces also in primates that are not yet phylogenetically evolved. Our immunohistochemical findings showed that the tested proteins have a different behaviour depending on the rank of baboons; in particular, in high-ranking baboons we found that all observed muscle fibres showed a significant staining pattern for SGs. On the contrary, in middle-ranking baboons, muscle fibres were positive in $85 \%$ of observations and in lowranking baboons only $55 \%$ of muscle fibres showed significant staining patterns, while the others were not positive. As is well known, cytoskeletal proteins form dynamic structures that respond to mechanical signals and other signals by undergoing short- or long-term changes in shape. ${ }^{25}$ Therefore, SGs are very important to transmit signals in order to regulate the mechanical forces required for movement and stabilization produced by actin and myosin. These forces are generated in sarcomeres and are transmitted longitudinally to myotendinous junctions and laterally to the extracellular matrix..$^{26,27}$ Our previous studies demonstrated that SGs and integrins may have an important function in reinforcing the arrangement of muscle fibres and recovering the signaling function of the entire membrane in order to restore contractile forces in the human masseter affected by a unilateral crossbite through a reorganization of the transmembrane proteins. ${ }^{20}$ In our opinion, the present data, obtained in the skeletal muscles of baboons unaffected by any motor diseases, could confirm a key role for SGs and integrins in the maintenance of contractile forces. The different staining patterns of these proteins observed in different ranks of baboons could be due to particular conditions of life of these primates. Human and non-human primates are characterized by two modes of social inter- action, called agonic and hedonic. The agonic mode, typical of Rhesus macaques and baboons, is based upon threat, power and anxiety; in this behavioural mode, primates follow a hierarchical organization and alpha-males are in a permanent state of alert to ensure that the others do not adopt an overpowering attitude. Conversely, chimpanzees are capable of establishing hedonic social relationships, which are more flexible, seeking collaboration and looking after themselves individually. Humans can switch between both modes. ${ }^{28}$ Male baboons disperse after maturity and, when joining a new group, they rapidly rise in rank. When they reach a high rank, they are more likely to engage in energy-intensive mate-guarding episodes. ${ }^{29,30}$ During these episodes, male foraging is constrained and consequently consorting males have feeding bouts that are 15\% shorter than those of nonconsorting males and may abandon consortships. ${ }^{29,31}$ Therefore, the gradual increase of SGs staining patterns, observed from low- to high-ranking baboons, can be due to an adaptation to a new animal behaviour. Indeed, the typical features of low-rank individuals, such as chronic stress and poor physical conditions, are associated with a greater risk of falling ill and slower healing, whereas high-rank conditions, such as elevated testosterone and intense reproductive effort, are associated with a lower risk of developing diseases and faster recovery. ${ }^{32}$ Consequently, these proteins, which play a key role in muscle activity by controlling contractile forces, may influence the phenotype of muscle fibres that determines the adaptation to new functional conditions. Therefore, in our opinion, we can hypothesize an intraspecies variation of the protein arrangement between the different ranks of baboons that affects their gradual adaptation to a different social status. This gradual adaptation could be confirmed by our observation of a significant staining pattern of SGs in the skeletal muscle of a baboon classified as middle-rank. In this case, it is possible that the subject is adapting to a higher rank and shows a change in the protein arrangement.This variation could imply an epigenetic modification of the muscular protein network which would cause a phylogenetic variation. Therefore, it is intriguing to explore whether the absence of SGs in several muscle fibres of low-ranking baboons can be due to the presence of alternative and unknown isoforms of these proteins that cannot yet be detected by the usual antibodies. This hypothesis, which might highlight the function of SGs in various social conditions as well as the different role of baboons within the same group, opens a new line of research that could lead to a better understanding of the real epigenetic mechanism at the basis of the evolution.

\section{References}

1. Campbell KP, Kahl SD. Association of dystrophin and an integral membrane glycoprotein. Nature 1989;338:259-62.

2. Yoshida M, Ozawa E. Glycoprotein complex anchoring dystrophin to sarcolemma. J Biochem 1990:108:748-52.

3. Ervasti JM, Campbell KP. A role for the dystrophin-glycoprotein complex as a transmembrane linker between laminin and actin. J Cell Biol 1993;122:809-23.

4. Campbell KP. Three muscular dystrophies: loss of cytoskeleton-extracellular matrix linkage. Cell 1995;80:675-9.

5. Straub V, Campbell KP. Muscular dystrophies and the dystrophin-glycoprotein complex. Curr Opin Neurol 1997;10:168-75.

6. Ozawa E, Noguchi S, Mizuno Y, Hagiwara Y, Yoshida M, 1998. From dystrophinopathy to sarcoglycanopathy: evolution of a concept of muscular dystrophy. Muscle Nerve 21:421-38.

7. Ozawa E, Mizuno Y, Hagiwara Y, Sasaoka T, Yoshida M. Molecular and cell biology of the sarcoglycan complex. Muscle Nerve 2005;32:563-76.

8. Anastasi G, Cutroneo G, Santoro G, Trimarchi F. The non-junctional sarcolemmal cytoskeleton: the costameres. Ital J Anat Embryol 1998;103:1-11.

9. Yoshida T, Pan Y, Hanada H, Iwata Y, Shigekawa M. Bidirectional signaling between sarcoglycans and the integrin adhesion system in cultured L6 myocytes. J Biol Chem 1998;273:1583-90.

10. Anastasi G, Cutroneo G, Trimarchi F, Santoro G, Bruschetta D, Bramanti P, et al. Evaluation of sarcoglycans, vinculin-talinintegrin system and filamin2 in $\alpha$ - and $\gamma$ sarcoglycanopathy: an immunohistochemical study. Int J Mol Med 2004;14:989-99.

11. Ervasti JM, Ohlendieck K, Kahl SD, Gaver MG, Campbell KP. Deficiency of a glycoprotein component of the dystrophin complex in dystrophic muscle. Nature 1990;345: 315-9.

12. Anastasi G, Cutroneo G, Santoro G, Arco A, Rizzo G, Bramanti P, et al. Costameric proteins in human skeletal muscle during muscular inactivity. J Anat 2008;213:28495.

13. Ohlendieck K, Campbell KP. Dystrophinassociated proteins are greatly reduced in skeletal muscle from mdx mice. J Cell Biol 1991;115:1685-94.

14. Ginjaar HB, van der Kooi AJ, Ceelie H, Kneppers AL, van Meegen M, Barth PG, et al. Sarcoglycanopathies in Dutch patients with autosomal recessive limb girdle muscular dystrophy. J Neurol 2000;247:524-9.

15. Calvo F, Teijeira S, Fernandez JM, Teijeiro 
A, Fernandez-Hojas R, Fernandez-Lopez $\mathrm{XA}$, et al. Evaluation of heart involvement in gamma-sarcoglycanopathy (LGMD2C). A study of ten patients. Neuromuscul Disord 2000;10:560-6.

16. Bönnemann CG, Wong J, Jones KJ, Lidov HGW, Feener CA Shapiro F et al. Primary $\gamma$ sarcoglycanopathy (LGMD 2C): broadening of the mutational spectrum guided by the immunohistochemical profile. Neuromuscul Disord 2002;12:273-80.

17. Dalkilic I, Kunkel LM. Muscular dystrophies: genes to pathogenesis. Curr Opin 2003;13:231-8.

18. Burkin DJ, Wallace GQ, Nicol KJ, Kaufman DJ, Kaufman SJ. Enhanced expression of the $\alpha 7 \beta 1$ integrin reduces muscular dystrophy and restores viability in dystrophic mice. J Cell Biol 2001;52:1207-18.

19. Anastasi G, Cutroneo G, Santoro G, Arco A, Rizzo G, Trommino C, et al. Integrins, muscle agrin and sarcoglycans during muscular inactivity conditions: an immunohistochemical study. Eur $\mathrm{J}$ Histochem 2006;50:327-36.

20. Cutroneo G, Piancino MG, Ramieri G,
Bracco P, Vita G, Isola G, et al. Expression of muscle-specific integrins in masseter muscle fibres during malocclusion disease. Int J Mol Med 2012;30:235-42.

21. Favaloro A, Speranza G, Rezza S, Gatta V, Vaccarino G, Stuppia L, et al. Muscle-specific integrins in masseter muscle fibres of chimpanzees: an immunohistochemical study. Folia Histochem Cytobiol 2009:47: 551-8.

22. Rak Y. The Australopithecine face. Academic Press, New York, USA; 1983.

23. Murthy KK, Salas MT, Carey KD, Patterson JL. Baboon as a nonhuman primate model for vaccine studies. Vaccine 2006;24:4622-4.

24. Langergraber KE, Prüfer K, Rowney C, Boesch C, Crockford C, Fawcett $\mathrm{K}$ et al. Generation times in wild chimpanzees and gorillas suggest earlier divergence times in great ape and human evolution. Proc Natl Acad Sci USA 2012;109:15716-721.

25. Ingber DE. Tensegrity: the architectural basis of cellular mechanotransduction. Annu Rev Physiol 1997;59:575-99.

26. Street SF. Lateral transmission of tension in frog myofibres: a myofibrillar network and transverse cytoskeletal connections are possible transmitters. J Cell Physiol 1983;114:346-64.

27. Monti RJ, Roy RR, Hodgson JA, Edgerton VR. Transmission of forces within mammalian skeletal muscle. J Biochem 1999;32:371-80.

28. Chance MRA, Jolly CJ. Social groups of monkeys, apes, and men. Dutton, NY, USA; 1970.

29. Rasmussen KLR. Changes in the activity budgets of yellow baboons (Papio cynocephalus) during sexual consortships. Behav Ecol Sociobiol 1985;17:161-70.

30. Gesquiere LR, Learn NH, Simao MC, Onyango P0, Alberts SC, Altmann J. Life at the top: Energetic and psychological stress in wild male primates. Science 2011;333: 357-60.

31. Alberts SC, Altmann J, WilsonML. Mate guarding constrains foraging activity of male baboons. Anim Behav 1996;51:1269-77.

32. Archie EA, Altmann J, Alberts SC. Social status predicts wound healing in wild baboons. Proc Natl Acad Sci USA 2012;109:9017-22. 